\title{
Estrés ante prácticas clínicas y su relación con habilidades \\ comunicativas en estudiantes de enfermeria de la Universidad \\ Nacional Jorge Basadre Grohmann - Tacna 2016
}

\author{
Stress before clinical \\ practices and its \\ relationship with \\ communication skills in \\ nursing students of the \\ National University Jorge \\ Basadre Grohmann - \\ Tacna 2016
}

\section{RESUMEN}

OBJETIVO: Determinar la relación entre el Stress y las habilidades Comunicativas de los estudiantes de Enfermería de la UNJBG 2016. MATERIAL Y MÉTODO: Estudio descriptivo, correlacional, transversal; con una muestra de 78 estudiantes. de enfermería, desde el 2 do año de estudios. Se aplicó el instrumento factores estresores (KEZKAK) y cuestionario sobre habilidades de la comunicación. Para el procesamiento de datos se utilizó el software SPSS versión 19, para realizar el análisis estadístico descriptivo y para establecer la relación entre las variables se utilizó la prueba estadística Chi cuadrado con un nivel de confianza de $95 \%$ y significancia sintótica de $<0,05$. RESULTADO: Se tiene que la mayoría son de 20 a 22 años de sexo femenino y 4 to año, con experiencia de práctica clínica mas de 5 veces, donde tuvieron momentos de preocupación el $94,9 \%$ y un nivel moderado de stress el $82,1 \%$, resaltando como factor estresante "Resultados de la evaluación de práctica". Se encuentra que el $74,4 \%$ tienen buena habilidad comunicativa. CONCLUSIÓN: A pesar de la experiencia en práctica clínica y buena habilidad comunicativa los estudiantes presentan moderada a alta incidencia de stress. No se encuentra relación significativa entre stress y habilidad comunicativa.

PALABRAS CLAVES: Estres clínica, habilidades comunicativas en estudiantes.

\section{Silvia Cristina Quispe Prieto ${ }^{1}$ Frida del Rosario Nuñez Sanchez ${ }^{2}$}

1. Doctora en Enfermería. Docente de la Universidad Nacional Jorge Basadre G. Magister en Tecnologìa Educativa, Especialista en Logopedia y Terapia Integral del Lenguaje

2. Lic. en Enfermería

\section{ABSTRACT}

OBJECTIVE: To determine the relationship between the Stress and the Communication skills of Nursing students of the UNJBG 2016. MATERIAL AND METHOD: Descriptive, correlational, cross-sectional study; with a sample of 78 nursing students, from the 2 nd year of studies. The instrument was applied stressors (KEZKAK) and questionnaire on communication skills. SPSS version 19 software was used for the data processing, to perform the descriptive statistical analysis and to establish the relationship between the variables, the Chi square statistical test was used with a confidence level of $95 \%$ and a synotic significance of $<0.05$. . RESULT: Most of them are 20 to 22 years old female and 4 th year, with clinical practice experience more than 5 times, where they had moments of concern $94.9 \%$ and a moderate level of stress $82.1 \%$, highlighting as a stressor "Results of the practice evaluation". It is found that $74.4 \%$ have good communication skills. CONCLUSION: Despite the experience in clinical practice and good communicative ability, students have moderate to high incidence of stress. There is no significant relationship between stress and communicative ability.

KEYWORDS: Clinical stress, communication skills in students. 


\section{INTRODUCCIÓN}

La Organización Mundial de la Salud define estrés como "conjunto de reacciones fisiológicas que preparan el organismo para la acción".(1) Alternativamente para precisar conceptos, se utiliza el término "respuesta de estrés" al referirse a la respuesta inespecífica del organismo a cualquier demanda, y el término de "estresor" o "situación estresante" referida al estímulo o situación que provoca una respuesta de estrés (1)

En estados Unidos, California, en una publicación de "Stress and the College Student" (el estrés y el estudiante universitario), encontró quede 1,6 millones de estudiantes del primer semestre el 30\% reportó frecuentes sentimientos de estar abrumado. Asimismo en el sitio web Campus Calm aduce que el estrés puede afectar el desempeño académico, citando la encuesta de Salud Mental y Estrés Universitario de 2009 administrada por Associated Press y MTV.

En conformidad a lo expresado todas las personas han experimentado en algún momento de su vida las consecuencias negativas que produce el stress y específicamente en su vida académica, en concreto los alumnos de pregrado de formación en carreras de la salud se enfrentan a situaciones de escenarios de práctica que demandan en ellos respuestas inmediatas, toma de decisiones basadas en sus habilidades cognitivas actitudinales y psicomotoras cuando acuden a los diferentes centros hospitalarios. Específicamente los estudiantes de la carrera profesional de enfermería presentan dentro de su plan de estudios la práctica clínica que representa un número considerado de horas donde acuden para su formación a diferentes escenarios de atención de salud, les permiten aplicar los conocimientos teóricos y desarrollar habilidades para la prestación de cuidados a los pacientes encontrándose expuestos a la sintomatología propia del stress. Las prácticas hospitalarias tienen un gran impacto educativo ya que los conocimientos que se adquieren en ellas permiten el desarrollo de las habilidades y actitudes propias de cada estudiante que les son necesarias para la adquisición de competencias y les permiten incorporarse como profesionales en el ámbito sanitario (2)

Por consiguiente desde ya el estudiante universitario presenta diversas fuentes de stress que van a influir directamente en su salud y su desempeño académico y se agrega su actuar directo del cuidado de los pacientes.Entre las situaciones vividas con más intensidad por los estudiantes de enfermería durante las prácticas asistenciales se encuentran: el sufrimiento, el contacto con enfermos terminales y el afrontamiento de la muerte, la relación con el enfermo y su familia entre otros. No obstante, las relaciones con los profesionales, los sentimientos de impotencia y la falta de conocimientos y destreza en las técnicas son los aspectos que más preocupan a los alumnos de enfermería (3). Es así que va demandar en él estudiante de enfermería el uso de diferentes estrategias de afrontamiento, teniendo el stress un alcance distinto según el estudiante, ya que dependerá de él de esa gama de estrategias y las habilidades con las que cuentan entre ellas la de comunicación que corresponde a ser una de las más importantes competencias del ser enfermero (1), representa una gran destreza para lidiar con asuntos difíciles. Crear una atmósfera de apertura no es una tarea fácil por lo que representa fundamental conocer como es el manejo de esta habilidad de los estudiantes de enfermería los cuales deben desarrollar esta competencia que le servirá por el resto de su vida profesional (4), por lo que se tuvo como objetivo determinar la relación entre el Stress y las habilidades Comunicativas de los estudiantes de Enfermería de la UNJBG,2016.

\section{MATERIAL Y MÉTODOS}

Estudio descriptivo, transversal, correlacional con muestra no probabilística, 78 estudiantesde enfermería desde el 2 do año de estudios.

Se aplicaron dos cuestionarios, uno fue de KEZKAK (5)que mide factores causantes de estrés en las prácticas clínicas de alumnos, se consideró como antecedente el estudio de Lopez $F(6)$ y se evalúa 12 factores stresores: 1 . Resultados de la evaluación de las prácticas. 2. Exceso-sobrecarga de trabajo. 3. Relaciones con familiares del paciente. 4. Riesgo a dañar al paciente. 5. Riesgo a sufrir contagio, daños o lesiones en la relación con el paciente. 6 . Implicaciones emocionales con el enfermo. 7. No saber controlar las relaciones con el paciente. 8 . Impotencia e incertidumbre ante situaciones determinadas. 9. Relaciones con los profesionales en las prácticas. 10. Contacto con el sufrimiento ajeno. 11. Desconocimiento ante una situación clínica. 12. Manejo de nuevas tecnologías. Sus respuestas en escala de Lickert del 1 al 5 El otro Cuestionario autoadministrado de Peña A.(7)para la medición de la comunicación interpersonal enfermera-paciente, consta de 17 ítemes en graduación en escala Lickert 1- 
5, construido con las variables: proactividad, actitud profesional, comunicación verbal, comunicación no verbal y apoyo emocional paraProactividad, Actitud profesional, Comunicación Verbal, No verbal y Apoyo Emocional.

Para realizar el análisis estadístico se utilizó el software SPSS, y se aplica la prueba Chi cuadrado con un nivel de confianza de $95 \%$ y significancia sintótica de $p<0,05$.

\section{RESULTADOS}

En el estudios se observa que de la muestra de 78 alumnos el 56,4\% tienen la edad entre 20 a 22 años, el $85.9 \%$ son de sexo femenino y el $43.6 \%$ son del 4 to año.(Tab №1)

Sepuede evidenciar que $71.8 \%$ de los estudiantes no es primera vez que acudieron a la práctica clínica, ya han acudido más de 5 veces, el $94.9 \%$ de estudiantes refieren que tuvieron momentos de preocupación medianamente. . (Tab №2)

En relación al stress ante las prácticas clínicas se tiene que el $82,1 \%$ de estudiantes de enfermería presentan un nivel Medio..(Tab №3)

Entre los Factores estresantes se halla en el estudio que el mayor porcentaje recae en los"resultados de la evaluación de prácticas", casi siempre el $43.6 \%$..(Tab №4)

En cuanto a las Habilidades Comunicativas en estudiantes de enfermería, el $74.4 \%$ presentan buena habilidad comunicativa. (Tab №5).

Se evidencia en el estudio el 59\% de estudiantes presentan un nivel moderado de Stress ante la práctica clínica y una buena habilidad comunicativa, No se encuentra relación significativa al aplicar la prueba estadística Chi cuadrado..(Tab №6)

\section{TABLA № 1}

Ccaracterizaciòn de la muestra en estudiantes de enfermería de la Universidad Nacional Jorge Basadre Grohmann. 2016

\begin{tabular}{lll}
\multicolumn{1}{l}{ CARACTERISTICAS } & $\mathbf{N}^{\circ}$ & $\%$ \\
EDAD & & \\
$17-19$ & 17 & 21,8 \\
$20-22$ & 44 & 56,4 \\
23 A MÁS & 17 & 21,8 \\
\hline
\end{tabular}

\begin{tabular}{|c|c|c|}
\hline SEXO & & \\
\hline FEMENINO & 67 & 85,9 \\
\hline MASCULINO & 11 & 14,1 \\
\hline \multicolumn{3}{|c|}{ AÑO DE ESTUdIOS } \\
\hline 2DO AÑO & 26 & 33,3 \\
\hline 3DO AÑO & 18 & 23,1 \\
\hline 4TO AÑO & 34 & 43,6 \\
\hline TOTAL & 78 & 100 \\
\hline
\end{tabular}

\section{TABLA № 2}

Aspectos relacionados a la practica clínica en los estudiantes de enfermería de la Universidad Nacional Jorge Basadre Grohmann. 2016

\begin{tabular}{|c|c|c|}
\hline CARACTERISTICAS & $\mathrm{N}^{0}$ & $\%$ \\
\hline \multicolumn{3}{|l|}{ PRIMERA VEZ LA PRACTICA } \\
\hline SI & 22 & 28,2 \\
\hline NO & 56 & 71,8 \\
\hline \multicolumn{3}{|l|}{ NÚMERO DE VECES DE PRÁCTICA } \\
\hline $3-5$ veces & 26 & 33,4 \\
\hline 5 a más veces & 52 & 67,9 \\
\hline \multicolumn{3}{|l|}{ TUVO MOMENTOS DE PREOCUPACIÓN } \\
\hline SI & 74 & 94,9 \\
\hline NO & 4 & 5,1 \\
\hline \multicolumn{3}{|c|}{ GRADO DE PREOCUPACIÓN PÒR SU PRÀCTICA } \\
\hline CASI NADA & 6 & 7,7 \\
\hline UN POCO & 13 & 16,7 \\
\hline MODERADAMENTE & 45 & 57,7 \\
\hline PREOCUPADO & 11 & 14,1 \\
\hline MUY PREOCUPADO & 3 & 3,8 \\
\hline TOTAL & 78 & 100 \\
\hline
\end{tabular}

\section{TABLA № 3}

Nivel de stress ante la practica clinica en estudiantes de enfermería de la Universidad Nacional Jorge Basadre Ggrohmann.2016

\begin{tabular}{|lcc|}
\hline \multicolumn{1}{|c}{ NIVEL DE STRESS } & $\mathrm{N}^{\circ}$ & $\%$ \\
\hline NIVEL DE STRESS & & \\
ALTO NIVEL & 12 & 15,4 \\
NIVEL MODERADO & 64 & 82,1 \\
BAJO NIVEL & 2 & 2,6 \\
TOTAL & 78 & 100,0 \\
\hline
\end{tabular}




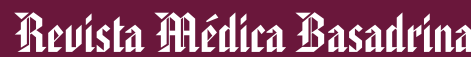

\section{TABLA № 4}

Factores stresantes en la practica clìnica en estudiantes de enfermería de la Universidad Nacional Jorge Basadre Grohmann.2016

\begin{tabular}{|c|c|c|c|c|c|c|c|c|c|c|c|c|}
\hline \multirow[t]{2}{*}{ FACTORES ESTRESANTES } & \multicolumn{2}{|c|}{ Nunca } & \multicolumn{2}{|c|}{ Rara Vez } & \multicolumn{2}{|c|}{ Alguna Vez } & \multicolumn{2}{|c|}{$\begin{array}{c}\text { Casi } \\
\text { Siempre }\end{array}$} & \multicolumn{2}{|c|}{ Siempre } & \multicolumn{2}{|c|}{ TOTAL } \\
\hline & $\mathbf{N}^{\circ}$ & $\%$ & $\mathbf{N}^{\circ}$ & $\%$ & $\mathbf{N}^{\circ}$ & $\%$ & $\mathbf{N}^{\circ}$ & $\%$ & $\mathbf{N}^{\circ}$ & $\%$ & $\mathbf{N}^{\circ}$ & $\%$ \\
\hline Resultados de la evaluación de prácticas & 1 & 1,3 & 10 & 12,8 & 27 & 34,6 & 34 & 43,6 & 6 & 7,7 & 78 & 100 \\
\hline Relaciones con familiares del Trabajo & 8 & 10,3 & 28 & 35,9 & 32 & 41,0 & 9 & 11,5 & 1 & 1,3 & 78 & 100 \\
\hline Riesgo a dañar al paciente & 9 & 11,5 & 20 & 25,6 & 30 & 38,5 & 13 & 16,7 & 6 & 7,7 & 78 & 100 \\
\hline $\begin{array}{l}\text { Riesgo a Sufrir contagio, daños o lesiones } \\
\text { en la relación conpaciente }\end{array}$ & 8 & 10,3 & 30 & 38,5 & 21 & 26,9 & 17 & 21,8 & 2 & 2,6 & 78 & 100 \\
\hline Implicaciones emocionales con el enfermo & 11 & 14,1 & 29 & 37,2 & 31 & 39,7 & 5 & 6,4 & 2 & 2,6 & 78 & 100 \\
\hline $\begin{array}{l}\text { No saber controlar las relaciones con le } \\
\text { paciente }\end{array}$ & 14 & 17,9 & 38 & 48,7 & 19 & 24,4 & 6 & 7,7 & 1 & 1,3 & 78 & 100 \\
\hline $\begin{array}{l}\text { Impotencia e incertidumbre ante situaciones } \\
\text { determinadas }\end{array}$ & 5 & 6,4 & 26 & 33,3 & 33 & 42,3 & 12 & 15,4 & 2 & 2,6 & 78 & 100 \\
\hline $\begin{array}{l}\text { Relaciones con los profesionales en las } \\
\text { prácticas }\end{array}$ & 7 & 9,0 & 23 & 29,5 & 33 & 42,3 & 14 & 17,9 & 1 & 1,3 & 78 & 100 \\
\hline Contacto con el sufrimiento ajeno & 6 & 7,7 & 27 & 34,6 & 33 & 42,3 & 12 & 15,4 & 0 & 0 & 78 & 100 \\
\hline Desconocimiento ante una situación clínica & 5 & 6,4 & 22 & 28,2 & 33 & 42,3 & 16 & 20,5 & 2 & 2,6 & 78 & 100 \\
\hline Manejo de nuevas tecnologías & 6 & 7,7 & 14 & 17,9 & 42 & 53,8 & 15 & 19,2 & 1 & 1,3 & 78 & 100 \\
\hline
\end{tabular}

\section{TABLA № 5}

Habilidades comunicativas en los estudiantes de enfermería de la Universidad Nacional Jorge Basadre Grohmann.2016

\begin{tabular}{llcc}
\multicolumn{1}{c}{ NIVEL DE STRESS } & No & $\%$ \\
\hline BUENA H & ABILIDAD COMUNICATIVA & 58 & 74,4 \\
REGULAR HABILIDAD COMUNICATIVA & 20 & 25,6 \\
TOTAL & 78 & 100 \\
\hline
\end{tabular}

\section{TABLA № 6}

Habilidades comunicativas en los estudiantes de enfermería de la Universidad Nacional Jorge Basadre Grohmann.2016

\begin{tabular}{|c|c|c|c|c|c|c|c|}
\hline \multirow[t]{2}{*}{$\begin{array}{l}\text { Nivel de } \\
\text { Stres }\end{array}$} & \multicolumn{2}{|c|}{$\begin{array}{c}\text { Buena } \\
\text { Habilidad } \\
\text { Comunicativa }\end{array}$} & \multicolumn{2}{|c|}{$\begin{array}{c}\text { Regular } \\
\text { Habilidad } \\
\text { Comunicativa }\end{array}$} & \multicolumn{2}{|c|}{ TOTAL } & $\begin{array}{c}\text { PRUEBA } \\
\text { CHI } \\
\text { CUADRADO }\end{array}$ \\
\hline & № & $\%$ & № & $\%$ & № & $\%$ & \\
\hline ALTO NIVEL & 11 & 14 & 1 & 1 & 12 & 15,38 & $p=0.26>0,05$ \\
\hline MODERADO & 46 & 59 & 19 & 25 & 65 & 83,33 & $\begin{array}{c}\text { No existe relación } \\
\text { estadística Significativa }\end{array}$ \\
\hline BAJO NIVEL & 1 & 1 & 0 & 0 & 1 & 1,28 & \\
\hline TOTAL & 58 & 74 & 20 & 26 & 78 & 100 & \\
\hline
\end{tabular}

\section{DISCUSIÓN}

En la presente investigación se tiene que la mayoría de estudiantes de enfermería (56.4\%) tienen entre 20 a 22 años y son, $85.9 \%$ de sexo femenino, resultados que se asemejan a los hallados en el estudio de Rivas A. Jiménez H. y otros (8)México 2,014, cuya muestra fue de 137 estudiantes de enfermería, determinaron que su edad promediofue de 21.6 años ( $D E=2.79 ; 18-37$ ), el $78.1 \%$ femenino y el $21.9 \%$ masculino. Por otro lado estos resultados se aproximan con los obtenidos por López V.(6)España 2011, en su estudio“Generadoras de estrés en los estudiantes de enfermería en las Prácticas Clínicas.", con 215 estudiantes de enfermería como muestra, reporta la media de edad global de 22 años. En general en nuestro país el predominio de cifras del sexo femeninono dista del sexo masculino(INEI), también se hallan datos respecto a la población universitaria el $6.8 \%$ de jóvenes que estudian enfermería son mujeres frente al $0,9 \%$ que son hombres.

La edad y sexo son variables importantes en esta etapa de vida; la edad determina en la persona la expectativa de comportamiento, adaptabilidad ante su contexto. Asímismo en la población en estudio, la profesión de 
enfermería a través de la historia se ha caracterizado por tener en su mayoría estudiantes del sexo femenino.

En relación a la frecuencia en que acuden a una práctica clínica evidencia que la mayoría $71.8 \%$ han acudido más de 5 veces; son de 4 to año (43.6\%) y el $94.9 \%$ refieren moderadamente momentos de preocupación. Estos resultados pueden ser respaldados por lo hallado en el estudio cualitativo de Rivera L.(9), Barcelona 2013, "Saber y experiencia de el/la estudiante de enfermería en sus prácticas de cuidado", con 11 estudiantes de enfermería como muestra, refiere en sus resultados que "el mundo hospitalario es un terreno desconocido e incierto para el/la estudiante de enfermería, en él se arriesga a perder el sentido de su competencia, su control y su propia confianza, de ahí que considere que se encuentra en un estado de vulnerabilidad". Los campos de práctica clínica proporciona oportunidades para el desarrollo de competencias del futuro profesional de enfermería por ello acuden a instituciones prestadoras de servicios de salud. La cognición del estudiante, aunque real, no tiene esencia palpable, al final se traducen en su quehacer práctico que no va separado del conocimiento teórico y práctico(10) por lo que experimentan ante este escenario una preocupación por el logro de los objetivos/ competencias en su formación.

En relación al stress ante las prácticas clínicas se tiene que el $82,1 \%$ de estudiantes de enfermería presentan un nivel Moderado. Estos resultados se asemejan al estudio de López F.(6) "Situaciones Generadoras de Estrés en los estudiantes de enfermería en las prácticas clínicas" obtiene que las puntuaciones medias obtenidas para los 12 ítems por curso, así como el nivel medio global de estrés. también se encuentra se semejanza con el estudio de Giménez J (11), España 2015 denominado "Estrés en estudiantes de enfermería durante sus prácticas clínicas en unidad de hospitalización a domicilio" en una muestra de 15 estudiantes, donde obtienen como resultado el nivel medio global de estrés que presentan en relación a cada una de las situaciones ponen de manifiesto que el $100 \%$ de las situaciones analizadas presentan puntuaciones medias. De acuerdo a los hallazgos estos y otros estudios similares se confirma que los estudiantes de enfermería presentan stress ante sus prácticas clínicas de una forma moderada en su gran mayoría, esta experiencia de formación práctica que está incluida en todos los currículos de formación a nivel nacional e internacional, constituye por su propia naturaleza y demanda de demostración y ejercicios de sus conocimientos teóricos una fuente de inseguridad sobre todo a los estudiantes que por primera vez acuden a la práctica que es lo que se espera como lo afirma, Rivas V. (8) precisa "debido a que por primera vez ingresan a una institución universitaria, la complejidad del proceso de adaptación y la falta de control sobre el nuevo ambiente, establece un cambio en el comportamiento para adaptarse al nuevo entorno",pero aún así se encuentra stress en estudiantes que incluso van por la quinta vez a un escenario de práctica, como es el caso de nuestro estudio, refrendado también lo manifestado por el mencionado investigador al precisar que los niveles de estrés percibidos por los estudiantes de enfermería son mayores que los de otras titulaciones.

En relación a los factores stresores se encuentra a "los resultados de la evaluación de prácticas" en Casi Siempre(43.6\%), hallazgos semejantes encontrados en la investigación de López V.(6)que destacan en mayor frecuencia el mencionado factor stresor. De manera global en nuestro estudio se puede analizar en factor stresante, casi siempre la preocupación por la evaluación de sus prácticas, es decir el puntaje de notas que pueden obtener al finalizar su práctica. Se encuentra que rara vez es referido como estresante el de "Riesgo a sufrir contagio, daños o lesiones en la relación con el paciente" y el factor enfocado a las relaciones interpersonales son la fuente de estrés que menos preocupa a los estudiantes. También se encuentra los otros estresores asociados y referidos como alguna vez son fuente de stress, los que tienen relación con la atención directa al paciente y manejo de emociones, estos resultados se asemejan a los hallados por Gimenez J. (11) que también presentan como situaciones estresantes a estos factores. Conforme se acumule experiencia en el quehacer del cuidado al paciente se espera que estos factores stresores vayan desapareciendo como fuentes de estrés.

En relación a habilidades Comunicativas el $74.4 \%$ de estudiantes de enfermería presentan buena habilidad comunicativa. Esta habilidad es competencia fundamental en la formación de la enfermera e implica desarrollar técnicas asociadas a la escucha activa, asertividad, empatía entre otros. Coincidiendo lo precisado con la investigación de Robles M (12) España 2013, en su "Estudio piloto sobre habilidades de comunicación y counseling en estudiantes universitarios" que tuvo como muestra 209 estudiantes, halló que los estudiantes de Enfermería perciben sus habilidades de la empatía y de reflexión 


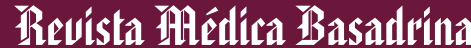

como más importantes que los estudiantes de Psicología.

La enfermera en el cuidado al paciente debe establecer una interacción donde es primordial la relación de ayuda y por supuesto que ello implica el tener que comunicarse, poder desarrollar esta habilidad comunicativa es preocupación de las instituciones formadoras de enfermeras toda vez que muchas veces no se pone en práctica, donde intervienen factores como son las características personales, la predisposición o las variables socioculturales.

En nuestro estudio se halla una buena habilidad de comunicativa lo que representa no solamente desde ya beneficioso para el paciente si no que va conducir a una relación recíproca donde también se beneficia el estudiante.

Al relacionar ambas variables del estudio se observa que un $59 \%$ de estudiantes presentan un nivel medio a alto de Stress ante la práctica clínica y una buena habilidad comunicativa, Al realizar la prueba Chi cuadrado no se halla significancia estadística. Estos hallazgos difieren a los resultados del trabajo de Leal C. (13).España, 2015“Las habilidades de comunicación como factor preventivo del síndrome de Burnout en los profesionales de la salud" con una muestra de 927 profesionales concluye unas adecuadas habilidades de comunicación incidirían en un menor padecimiento del síndrome de Burnout.

La importancia que tiene el entrenar a los profesionales en la adquisición de habilidades de comunicación, aumenta el peso que la calidad de las relaciones interpersonales (interacción humana) y las características individuales tienen en el cuidado de enfermería. Como lo menciona Leal C. (13), refiere que de ese modo se contribuiría a mejorar la salud de los trabajadores en el ámbito clínico. Por otro lado no debemos olvidar que, como ponen de manifiesto algunos estudios, unas adecuadas habilidades de comunicación del profesional de salud tienen su impronta en la satisfacción, reducción del estrés, adherencia al tratamiento y el sufrimiento de los paciente. Concluyendo que los estudiantes de enfermería enfrentan momentos de preocupación ante sus prácticas clínicas y en su mayoría presentan stress moderadamente, entre los factores generadores de stress casi siempre son los "Resultados de la evaluación de práctica", asimismo presentan la mayoría buena habilidad comunicativa. Las variables en estudio no presentan relación estadística significativa. Por la importancia que reviste la formación integral de los futuros profesionales de enfermería, se recomienda establecer programas de formación en estrategias para reducir el estrés, como también fortalecer el sentido pedagógico de la evaluación de las prácticas clínicas.

\section{Agradecimientos y Conflictos de Intereses.}

El agradecimiento a Los estudiantes que colaboraron en el estudio por su buena disposición y colaboraciónpara la presente investigación 


\section{REFERENCIAS BIBLIOGRÁFICAS}

1. Fernández G, Jiménez $M$. Control del estrés. UNED Ediciones. Madrid. 2002

2. Pagés J, Blanco R, Cónsul G, ZF. Aprendizaje y satisfacción de los estudiantes de enfermería en las prácticas clínicas (II). Atención Primaria de Salud. Enferm Clín. 1998; 7 (1): 16-24

3. López MA, Sánchez V. Percepción del estrés en estudiantes de enfermería en las prácticas clínicas. Enferm Clínica (Revista Electrónica) 2005; 15 (6): 307-313. Disponible en URL:http://www.elsevier.es/es/revistas/enfermeria-clinica35/percepcion-estres-estudiantes-enfermeria-las-practicasclinicas-13082986-originales-2005.

4. Stora B. ¿Qué sé acerca del estrés?. Publicaciones cruz $1^{\circ}$ Edición México Df.. 1991

5. Gorostidi X, Uranga MJ, Alberdize MJ, Barandiaran M. KEZKAK: Cuestionario bilingüe de estresores de los estudiantes de enfermería en las prácticas clínicas. Gac Sanit. 2003; 17 (1):37-50

6. López V Francisca, López M María José. Situaciones generadoras de estrés en los estudiantes de enfermería en las prácticas clínicas. Cienc. enferm. [Internet]. 2011; 17(2): 47-54. Disponible en:

http://www.scielo.cl/scielo.php?script=sci_arttext\&pid=S0 717-95532011000200006\&lng=es. http://dx.doi.org/10.4067/S0717-95532011000200006

7. Peña A. Evaluación de competencias comunicativas de especialistas en Imagenología. Educación Médica Superior [revista en Internet]. 2016 Disponible en: http://www.ems.sld.cu/index.php/ems/article/view/682

8. Rivas V, Jiménez C, Cruz M y otros. Frecuencia e Intensidad del Estrés en Estudiantes de Licenciatura en Enfermería de la DACS. Rev Horizonte Sanitario [online].2014.Vol.13 issn 2007.

9. Rivera L. Saber y experiencia de el/la estudiante de enfermería en sus prácticas de cuidado. Tesis Universidad de Barcelona 2013

10. Bettancourt L, Muñoz L, y otros. El docente de enfermería en los campos de práctica clínica: un enfoque fenomenológico. Rev. Latino-Am. Enfermagem Artículo Originale 19(5): [09 pantallas] sep.-oct. 2011 www.eerp.usp.br/rlae

11. Giménez J. "Estrés en estudiantes de enfermería durante sus prácticas clínicas en unidad de hospitalización a domicilio" Universidad de Alicante, 2015

12. Robles M. Estudio piloto sobre habilidades de comunicación y counseling en estudiantes universitarios. Boletín de Psicología, No. 107, Marzo 2013, 7-19

13. Leal C., Díaz J. Tirado S., Rodríguez J. et. Las habilidades de comunicación como factor preventivo del síndrome de Burnout en los profesionales de la salud. Anales Sis San Navarra [Internet]. 2015 38(2): 213-223. Disponible en: http://scielo.isciii.es/scielo.php?script=sci_arttext\&pid=S11 37-66272015000200005\&lng=es. http://dx.doi.org/10.4321/S1137-66272015000200005

\section{Correspondencia}

Silvia Quispe Prieto

Silviaq24@hotmail.com
Fecha de recepción: 19 de mayo de 2017

Fecha de aceptación: 20 de junio de 2017 